\title{
A HAPTIC DISPLAY FOR ROBOTIC REHABILITATION OF STROKE
}

\author{
PRADEEP NATARAJAN ${ }^{1}$, WEN LIU ${ }^{2}$, JOSH OECHSLIN ${ }^{1}$, and ARVIN AGAH ${ }^{1}$ \\ ${ }^{1}$ Dept. of Electrical Engineering and Computer Science, University of Kansas, Lawrence, KS \\ ${ }^{2}$ The University of Kansas Medical Center, Kansas City, KS
}

\begin{abstract}
The effects of stroke are debilitating on the American population. Past studies of robot-aided motor training for survivors have proven to be effective in upper limb motor recovery. However, survivors also suffer from loss or impairment of sensation. Sensory impairment is an important predictor for motor recovery of stroke survivors. Studies have suggested that sensory inputs during robot-aided motor training might be critical for the creation and promotion of cortical reconstruction due to brain plasticity during post-stroke recovery. This paper presents a new haptic display for the handle of the InMotion2 robot in order to enhance cutaneous sensory inputs for stroke survivors during hand motion. The sensory enhancement is realized through pins attached to servomotors mounted inside the robot handle that vibrate and contact the middle and index fingers, the palm, and the thumb during motor training. Each servomotor is independently controlled using a computer via parallel port with a Field-Programmable Gate Array (FPGA) board as the hardware interface.
\end{abstract}

KEYWORDS: Rehabilitation Robots, Haptic Display, Biomedical Robots, Stroke.

\section{INTRODUCTION}

Every 45 seconds, someone in the United States has a stroke. Stroke killed an estimated 163,538 people in 2001 and is the nation's third leading cause of death, ranking just behind diseases of the heart and all forms of cancer. Stroke is a leading cause of serious, long-term disability in the United States. Each year more than 700,000 Americans suffer a stroke, of which about 500,000 are first attacks (200,000 recurrent attacks). From the early 1970s to early 1990s, the estimated number of non-institutionalized stroke survivors increased from 1.5 to 2.4 million and an estimated 4.7 million stroke survivors are alive today. In 2004, the estimated total of direct and indirect cost of stroke is $\$ 53.6$ billion [1]. Approximately $50 \%$ to $60 \%$ of stroke survivors are moderately or minimally impaired, who may greatly benefit from rehabilitation $[2,3,4,5]$. Loss of arm function is perceived as a major problem by the majority of chronic stroke patients [6]. Up to 3.1 million stroke survivors have a loss of protective, proprioceptive, and touch sensation [7, 8]. There has been a strong demand from patients and caregivers to develop effective treatments to improve sensorimotor function of hemiparetic arm/hand for stroke survivors to improve the patients' quality of life and to reduce socioeconomic impact of the disease-induced disability.

Studies have shown that activity-dependent cortical plasticity, which is the reorganization of the cerebral cortex of the brain, accompanies motor learning; and that rehabilitation and training influence the pattern of reorganization $[9,10]$. Consequently, several treatment approaches have been introduced to improve sensorimotor function, including muscle strength training $[11,12]$, task-specific practice $[13,14]$, forced use of the paretic limb by restraining the contra-lateral limb $[15,16]$, and robot-aided motor training $[17,18,19,20]$. Studies of robot-aided motor training have shown that it is more productive for patient treatment and more effective for functional improvement of the paretic limb after training rather than conventional physical therapy [21]. 
Although robot-aided motor training has been proven to be more effective than conventional physical therapy for functional improvement of stroke patients' paretic limbs, patients can encounter great difficulty in sensing hand contact with the robot handle. Because the patients have diminished sensation, the probability of cortical reorganization and motor recovery decreases. Past studies have shown that sensory impairment is a predictor for the motor recovery of stroke patients [6, 22]. Moreover, sensory inputs during motor training could be a source of critical triggering signals that evokes and promotes cortical plasticity [7]. In order to improve the effectiveness of robot-aided motor training, the addition of haptic displays, i.e., enhanced cutaneous sensory inputs, may increase sensory feedback for stroke patients (critical for motor recovery). By adding enhanced cutaneous sensory inputs to the handle of the InMotion2 robot, stroke patients may receive more effective robot-aided motor training. The design of the haptic display requires cutaneous sensory input applied to the palmar side of the middle and index fingers, the thumb, and the palm of the patient. It has been shown that an indentation of $0.002 \mathrm{~m}$ to those fingers and palm is optimal [23]. Studies have shown that vibration stimuli of around $30 \mathrm{~Hz}$ can be sensed by human skin [24]. The robot-aided motor training program should be able to adjust the amplitude and the frequency in real time if the patient is not able to feel the vibration.

This paper presents the design and implementation of a cost-effective sensory-enhanced handle that uses radio control servomotors to produce the cutaneous sensory inputs. The servomotors can be controlled by the same computer that controls the InMotion2 robot [25]. This design has been validated through the fabrication of a prototype handle.

\section{HARDWARE}

The InMotion2 robot is based on the patented MIT-MANUS technology and is commercially available from Interactive Motion Technologies, Inc. [25]. This robot (Figures 1a and 1b) is a novel tool for teaching and therapy in manual and manipulative skills. It is capable of safely "shaping" motor skills - a machine implementation of "hand-over-hand" instruction [25]. An interactive robotic therapist interacts with a patient to shape the motor skills of the patient by guiding the patient's limb through a series of desired exercises with a robotic arm. The patient's limb is brought through a full range of motion to rehabilitate multiple muscle groups [26]. This robot has been effectively tested at Burke Rehabilitation Hospital in White Plains, NY [27].

The size of the servomotor was one of the main criteria in choosing the appropriate servomotor for the haptic display. The servomotors were required to fit inside a cylindrical handle with a minimum diameter with appropriate angular velocity and torque. The MX-50HP/BB, manufactured by Maxx Products International, was chosen and used. This is a high performance servomotor (Figure 1c) that combines low weight, small size, high speed and appropriate torque with low cost. Each servomotor is slightly larger than a quarter, rotates 60 degrees in 80 milliseconds, and produces 0.18 Newton-meter of torque [28].

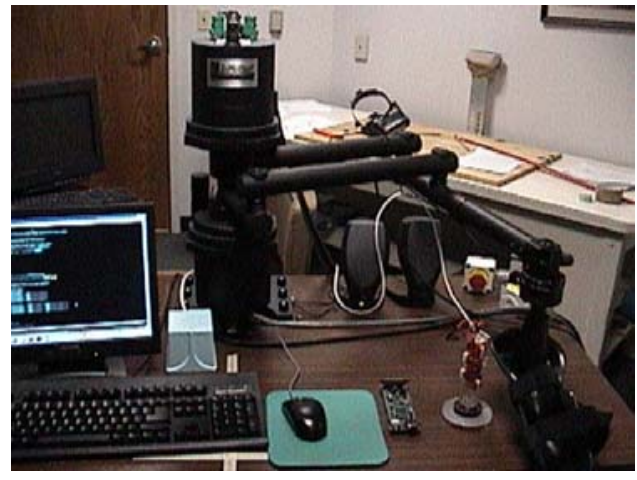

(a)

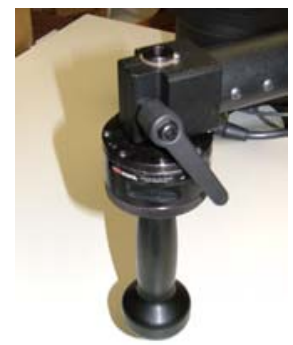

(b)

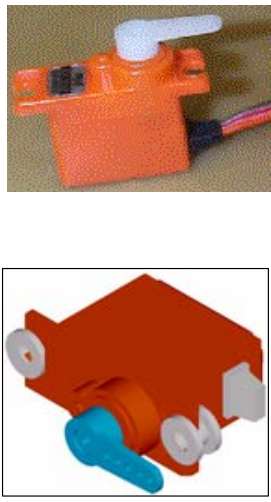

(c)

Figure 1. (a) The rehabilitation robot, (b) the robot's handle, and (c) servomotor and its model. 
In order to produce a displacement of $0.002 \mathrm{~m}$ (the amplitude required for the vibration), an arm that is $0.01 \mathrm{~m}$ in length (calculated from the center of the rotating shaft) has to rotate about 6 degrees. Since the angle of rotation is small, it can be simplified as a vertical motion for calculation purposes. The peak speed of the servomotor is given as $80 \mathrm{~ms}$ per 60 degrees. Hence for an angular displacement of about 6 degrees, theoretically it should take only $8 \mathrm{~ms}$. However this calculation does not consider the slew rate. It has been found that both the $10 \%$ to $90 \%$ rise time and the $90 \%$ to $10 \%$ fall time was $41 \mathrm{~ms}$ for a $0.002 \mathrm{~m}$ displacement [28]. As the amplitude is increased, the speed will be largely limited by the slew rate. For smaller amplitudes it is possible to produce frequencies close to $30 \mathrm{~Hz}$ which is acceptable in this study. In order to transfer deformation to the skin, a $0.001 \mathrm{~m}$ diameter steel wire was used to fabricate mechanical pins. Each pin was bent at the required hole on the plastic arm at a right angle. Sorbothane, a proprietary viscoelastic and polymeric solid manufactured by Ergotech Protective Devices Inc., was selected to be placed around the external cylindrical enclosure. This sheet of Sorbothane would act as a vibration filter by eliminating the undesirable vibration. Ergotech sells handle covers to reduce fatigue and undesirable vibration with a thickness of 1/10" and bonded with a non-slip Toughtek cover. DesignCAD 3D MAX Version 12.0.023 was used to create accurate and technical drawings for the handle design in order to evaluate different options (Figure 1c).

In the prototype handle, the servos are mounted and glued onto a high density plastic cuboid that has grooves cut into it to fit the servos. This single piece of plastic holds the servos in place. High density plastic is chosen due to its ease of machining. The arrangement is then enclosed in an external cover, which is also made of high density plastic. In the final design, holes will be drilled into the external cover to enable the steel pins to protrude out just enough to produce the necessary cutaneous sensory input. The arrangement in Figures $2 \mathrm{a}$ and $2 \mathrm{~b}$ shows five servomotors clearly and one at the bottom that is only partially visible. The servos are numbered 0 to 5 . The design requirement was to have a minimum of four servos in the handle but six servos were used to show the scalability of the design. Thus more sensory inputs could be given to the palm of the patient, if required. Human factors were important design constraints for the servo arrangement. The handle is designed in such a way that it avoids radial and ulnar deviation, and wrist flexion and extension. If a patient was forced to hold the robot handle during a training session such that there was radial deviation, the patient would experience unnecessary pain after the session.

\section{SOFTWARE}

One of the main advantages of using an off-the-shelf servomotor is that it is simple and easy to control. It includes a motor, gearbox, in-built position feedback mechanism and controlling electronics. The servomotors have three a wire interface, one for the positive of the power supply, one for the ground/negative and one for the control signal. The servomotors are powered by an external $4.5 \mathrm{~V}$ power supply. Each servomotor can be controlled to move to any position by using pulse width modulation. The control pulse is a positive going pulse with 'On' time of $0.7 \mathrm{~ms}$ to $2.3 \mathrm{~ms}$ followed by 'Off' time of $12 \mathrm{~ms}$ to $20 \mathrm{~ms}$. Thus the time period of one whole pulse is about $14 \mathrm{~ms}$. During the 'On' time the pulse is high (3V to $5 \mathrm{~V})$ and during the 'Off' time the pulse is low (0V). For this particular servomotor, sending $0.7 \mathrm{~ms}$ 'On' time pulses sets the servo to one end position and sending $2.3 \mathrm{~ms}$ pulses sets it to the other end position. Sending $1.5 \mathrm{~ms}$ pulses sets the servo motor to the center position. The total angular displacement of the arm of this servomotor is measured to be about 185 degrees.

Although controlling the servomotor is relatively simple and commercial servo control chips are easily available, none of them provide the flexibility and the features required for this application. They are either limited by the number of servomotors that can be controlled at a time or the speed at which the control can be switched between the servos. The sensory-enhanced handle requires the controlling of the frequency and amplitude of each servomotor independently at all times. Hence a custom control is desired for this application. A custom control similar to the one used in [28] is implemented. The specific platform selected for this purpose is the XSA-50, 
manufactured by XESS Corp. This is a development platform available off-the-shelf that contains a Xilinx@ Spartan-II Field-Programmable Gate Array (FPGA), flash memory and a $100 \mathrm{MHz}$ onboard programmable clock. Using a FPGA with 50,000 gates makes the design extremely scalable and flexible which is difficult to achieve by using the commercial servo controllers. The FPGA is programmed using Very High Speed Integrated Circuit Hardware Description Language (VHDL). The digital design consists of two main components - a decoder implemented as a Finite State Machine (FSM) and a programmable pulse generator. The decoder is used for selecting the specific servomotor and the pulse generator produces the required control signal to be sent to the servomotor. The XSA-50 platform is connected to the computer through the parallel port (Figure 2c), of the same computer that is used to control the InMotion2 robot. Six out of the eight data pins of the parallel port are used to send signals from the computer to the XSA-50 platform. Since four bits are used to select a servomotor, the software component of the sensoryenhanced handle can be scaled up to control 16 servomotors without any major modification. The current design of FPGA-based controller provides 4 bit resolution for the amplitude. For additional resolution, the unused pins from the parallel port can be used. The programmable pulse generator is an improvised version of a down counter. The on-board clock is set at a frequency of $50 \mathrm{MHz}$. This frequency is divided by 1024 within the VHDL program giving a new clock frequency of $48.83 \mathrm{kHz}$. This is used as the clock input for the pulse generator. The 'Off' time of the pulse is a constant and it is set at $13 \mathrm{~ms}$, a safe value as the 'Off' time should be at least above $12 \mathrm{~ms}$. If this time is too small, then the servomotor arm will not have enough time to rotate to the opposite side of the initial motion. Since the required motion is vibratory, the arm of the servomotor is set at its midpoint and from there an angular displacement of \pm 6 degrees is applied. This angular displacement is converted in terms of time period as $\pm 0.14 \mathrm{~ms}$ from the midpoint. The servomotor can be rotated to its midpoint by applying a pulse with 'On' time of about $1.5 \mathrm{~ms}$. This time period is equal to 73 pulses at the clock frequency of $48.83 \mathrm{kHz}$. Similarly $0.14 \mathrm{~ms}$ is approximately equal to 7 clock pulses. A down counter is implemented, initially at 73 . If the counter starts from 73-7=66, then the servomotor arm will move in one direction. In the subsequent cycle when the counter starts from $73+7=80$, then the servomotor arm will move in the opposite direction. The amplitude can be increased by increasing the number to be added or subtracted from 73. But due to the slew rate, increasing the amplitude will result in the reduction of the frequency of vibration. Figure 2 shows the prototype handle connected to the XSA-50 platform using separate single-lead wires. This connection will be replaced by ribbon cables in the final design. This type of connection was preferred for the prototype as it was easy to debug. The XSA-50 board and all the servomotors are connected to a breadboard for ease of debugging.

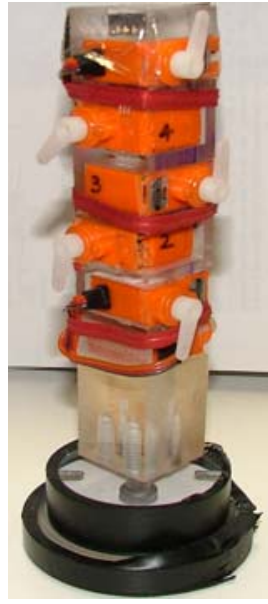

(a)

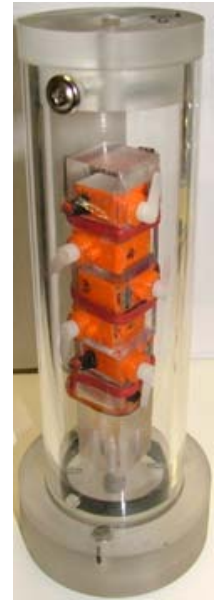

(b)

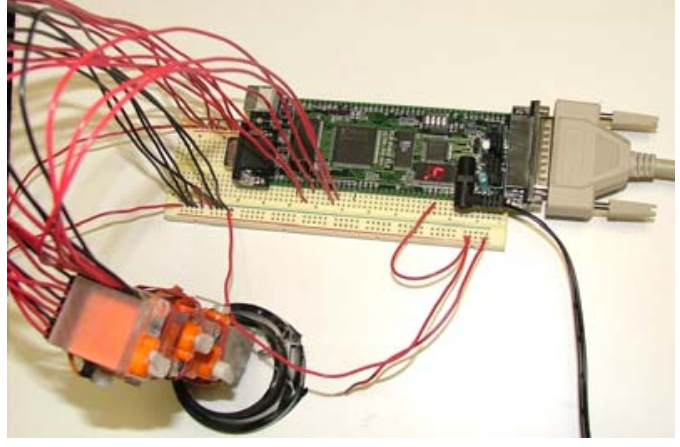

(c)

Figure 2. (a) The designed handle, (b) the handle with the cover, and (c) the control system. 
During the robot-aided training, various servomotors will be actuated depending on the movement of the robot arm. The computer that controls the InMotion2 robot is a natural choice to control the servomotors too. Since controlling the servomotors is not processor intensive, running the servo control program in the same computer doe not cause any hindrance to the robot-aided training programs. This makes it easier to integrate the sensory-enhanced handle with the InMotion2 robot both in terms of hardware and software. The robot controller runs on the Linux operating system. The robot controller is developed using $\mathrm{C}$ programming language and the user interface is created using Tcl/TK. The servo control software is also written in C programming language. To start and to stop any servomotor a series of five signals are sent to the parallel port. As far as the software is concerned it is simple output calls to the parallel port. Each parallel port output call only takes about $1 \mu$ sec.

\section{CONCLUSION}

The proposed experiment is aimed at developing a new stroke rehabilitation program to facilitate sensory inputs of a haptic display with robot-aided motor training, and to prove the concept that sensory enhancement integrated with motor training can further improve sensorimotor function for hemiparetic upper limbs of stroke patients. A total of 40 stroke patients with sensorimotor deficits will be recruited and randomly assigned to an experimental or a control group. Subjects in the experimental group will undergo robot-aided training with enhanced cutaneous sensory inputs. Subjects in the control group will be trained in the same motor tasks, but without enhanced sensory inputs. Subjects in both groups will be evaluated for their sensory and motor function in a baseline, an end-treatment, and follow-up tests. The effectiveness of

enhanced sensory inputs for motor recovery will be assessed through the comparison of outcomes of two training programs.

\section{ACKNOWLEDGEMENTS}

This project is partially supported by research grants from NIH (NS043331) and NSF (BES0302466).

\section{REFERENCES}

[1] American Stroke Association, http://www.strokeassociation.org, December 2005.

[2] M.L. Dombovy, "Rehabilitation and the course of recovery after stroke," in J. Whisnant (Ed.) Stroke population, cohorts and clinical trials, Butterworth-Heinemann, Boston, 1993.

[3] J.P. Broderick, T.F. Phillips, J.P. Whisnant, W.M. O’Fallon, and E.J. Bergstralh, "Incidence rates of stroke in the eighties: The end of the decline in stroke," Stroke, vol. 20, 577-582, 1989.

[4] H.S. Jorgensen, H. Nakayama, H.O. Raaschou, J. Vive-Larsen, M. Stoier, and T.S. Olsen, "Outcome and time course recovery in stroke. Part I and II: The Copenhagen stroke study," Archives of Physical Medicine and Rehabilitation, vol. 76, pp. 406-412, 1995.

[5] D.T. Wade, and R.L. Hewer, "Functional abilities after stroke: Measurement, natural history and prognosis," Journal of Neurology, Neurosurgery and Psychiatry, vol. 50, pp. 177-182, 1987.

[6] J.G. Broeks, G.J. Lankhorst, K. Rumping, A.J. Prevo. "The long-term outcome of arm function after stroke: results of a follow-up study," Disability and Rehabilitation, vol. 21, no. 8, pp. 357-364, 1999.

[7] J.S. Feigenson, M.L. McCarthy, S.D. Greenberg, and W.D. Feigenson, "Factors influencing outcome and length of stay in a stroke rehabilitation unit, Part 2,” Stroke, vol. 8, 657-62, 1977.

[8] J. Boivie, G. Leijon, and I. Johansson, "Central post-stroke pain - a study of the mechanisms through analyses of the sensory abnormalities,” Pain, vol. 37, No. 2, pp. 173-185, 1989.

[9] W.M. Jenkins, M.M. Merzenich, M.T. Oches, T. Allard, and E. Guic-Robles, "Functional reorganization of primary somatosensory cortex in adult owl monkeys after behaviorally controlled tactile stimulation,” Journal of Neurophysiology, vol. 63, pp. 82-104, 1990.

[10] G.H. Recanzone, W.M. Jenkins, and M.M. Merzenich, "Progressive improvement in discriminative abilities in adult owl monkeys performing a tactile frequency discrimination task," Journal of Neurophysiology, vol. 67, pp. 1015-1030, 1992. 
[11] P.W. Duncan, and M.B. Badke, Stroke Rehabilitation: Recovery of Motor Control, New York Book Publishers, New York, 1987.

[12] C. Guiliani, "Strength training for patients with neurological disorders," Neurology Report, vol. 19, pp. 29-34, 1995.

[13] F. Malouin, M. Potvin, J. Prevost, C.L. Richards, and S. Wood-Dauphinee, "Use of intensive task-oriented gait training program in a series of patients with acute cerebrovascular accidents," Physical Therapy, vol. 72, pp. 781-793, 1992.

[14] L. Ada, and P. Westwood, "A kinematic analysis of recovery of the ability to stand up following stroke,” Australian Journal of Physiotherapy, vol. 38, pp. 135-142, 1992.

[15] S.L. Wolf, D.E. LeCraw, L.A. Barton, and B.B. Jann, "Forced use of hemiplegic upper extremities to reverse the effect of learned nonuse among chronic stroke and head-injured patients,” Experimental Neurology, pp. 104-125, 1989.

[16] W.H. Miltner, H. Bauder, M. Sommer, C. Dettmers, and E. Taub, "Effects of constraint induced movement therapy on patients with chronic motor deficits after stroke: A replication," Stroke, vol. 74, pp. 347-354, 1999.

[17] D.J. White, A.M. Schneider, and W.K. Brogan Jr., "Robotic orthosis for stroke patient rehabilitation," in Proceedings of the 15th Annual International Conference on IEEE Engineering in Medicine and Biology, pp. 1272-1273, San Diego, CA, 1993.

[18] P.S. Lum, D.J. Reinkensmeyer, and S.L. Lehman, "Robotic assist devices for biannual physical therapy: preliminary experiments," IEEE Transactions on Rehabilitation Engineering, vol. 1, pp. 185-191, 1993.

[19] H.I. Krebs, M.L. Aisen, B.T. Volpe, and N. Hogan, "Robot-aided neuro-rehabilitation: initial application to stroke rehabilitation," in Proceedings of the Second International Symposium on Medical Robotics and Computer Assisted Surgery, John Wiley \& Sons, November 1995.

[20] M.L Aisen, H.I. Krebs, N. Hogan, F. McDowell, and B.T. Volpe, "The effect of robotassisted therapy and rehabilitative training on motor recovery following stroke," Archives of Neurology, vol. 54, pp. 443-446, 1997.

[21] C.G. Burgar, P.S. Lum, M. Shor, and H.F.M. Van der Loos, "Rehabilitation of upper limb dysfunction in chronic hemiplegia: robot-assisted movements vs. conventional therapy," Archives of Physical Medicine and Rehabilitation, vol. 80, pp. 1121, 1999.

[22] A. Kusoffsky, I. Wadell, and B.Y. Nilsson, "The relationship between sensory impairment and motor recovery in patients with hemiplegia," Scandinavian Journal of Rehabilitation Medicine, vol. 14, no. 1, pp. 27-32, 1982.

[23] R.S. Fearing, G. Moy, and E. Tan, "Some basic issues in teletaction," in Proceedings of the IEEE International Conference on Robotics and Automation, vol. 4, pp. 3093-3099, Albuquerque, NM, April 1997.

[24] W. Liu, L. Lipsitz, M. Montero-Odasso, J. Bean, C. Kerrigan, and J.J. Collins, "Overcoming age-related sensory loss with mechanical noise," Archives of Physical Medicine and Rehabilitation, vol. 83, pp. 171-176, 2002.

[25] N. Hogan, H.I. Krebs, J. Charnnarong, P. Srikrishna, and A. Sharon, "MIT-MANUS: A workstation for manual therapy and training I," in Proceedings of the IEEE International Workshop on Robot and Human Communication, pp. 161-165, Tokyo, September 1992.

[26] N. Hogan, H.I. Krebs, and J. Charnnarong, "Interactive robotic therapist," US patent 5,466,213, Massachusetts Institute of Technology, 1995.

[27] H.I. Krebs, N. Hogan, M.L. Aisen, and B.T. Volpe, “Robot-aided neurorehabilitation,” IEEE Transactions on Rehabilitation Engineering, vol. 6, no. 1, pp. 75-87, 1998.

[28] C.R. Wagner, S.J. Lederman, and R.D. Howe, "A tactile shape display using RC servomotors," in Proceedings of the 10th International Symposium on Haptic Interfaces for Virtual Environment and Teleoperator Systems, pp. 354-355, Orlando, March 2002. 